\title{
Comparative analysis of zooplankton diversity in freshwaters: What can we gain from metagenomic analysis?
}

4 Authors: Marie-Ève Monchamp ${ }^{1-2-a,}$ David A. Walsh ${ }^{2-3}$, Rebecca E. Garner ${ }^{2-3}$, Susanne A. Kraemer ${ }^{2-3}$,

5 Beatrix E. Beisner ${ }^{2-4}$, Melania E. Cristescu ${ }^{1-2}$, Irene Gregory-Eaves ${ }^{1-2}$

$6{ }^{1}$ Department of Biology, McGill University, Montreal, Quebec, Canada;

$7 \quad 2$ Groupe de recherche interuniversitaire en limnologie (GRIL);

$8{ }^{3}$ Department of Biology, Concordia University, Montreal, Quebec, Canada;

$9{ }^{4}$ Department of Biological Sciences, University of Quebec at Montreal, Montreal, Quebec, Canada.

10 a correspondence: Marie-Ève Monchamp me.monchamp@gmail.com

11 Department of Biology, McGill University

12 Stewart Biology Building, Room N4/19

131205 Docteur Penfield, Montreal, Quebec, H3A 1B1, Canada

Running head: Zooplankton estimation via shotgun metagenomics

Keywords: Shotgun sequencing; Environmental DNA (eDNA); Freshwater ecology; Biodiversity survey; Ecological assessment

\section{Author contributions}

21 M-ĖM, IGE, MEC and DAW designed the study. REG and SAK made major contributions to the

22 acquisition and interpretation of the metagenomic data. BEB provided the morphological zooplankton data. M-ÈM, SK, and REG performed data analysis and produced the figures. M-ÈM wrote the first draft of the manuscript and all authors contributed to editing the manuscript.

\section{Acknowledgements}

27 We thank the NSERC- funded LakePulse Network (2017-2019), Yannick Huot for leading LakePulse,

28 Marie-Pierre Varin and Maxime Fradette for coordinating sampling and lab work. We are grateful to Vera

29 Onana (Concordia University) for her contribution to producing some of the molecular data. We

30 additionally thank Cindy Paquette (UQAM) for providing curated zooplankton identification and count

31 tables, and Michelle Gros (McGill University) for the rotifer identification and help compiling zooplankton

32 data. We also wish to acknowledge the contribution of the high-throughput sequencing platform of the

33 McGill University and Genome Québec Innovation Centre (Montreal, Canada). M-ÈM received a

34 postdoctoral fellowship co-funded by the Groupe de recherche interuniversitaire en Limnologie (GRIL),

35 Liber Ero, and McGill University. The project was supported by LakePulse. IG-E, DAW and MEC

36 acknowledge additional funding from the Canada Research Chairs program and NSERC Discovery

37 program. The authors declare no conflict of interest. 


\section{Abstract}

39 Molecular genetic approaches applied to environmental DNA have great potential for biodiversity

40 research and ecosystem monitoring. A metagenome contains genetic information from all organisms

41 captured in an environmental sample. It has been primarily used to study bacteria and archaea, but

42 promising reports focusing on metazoan diversity are emerging. However, methodological uncertainties

43 remain, and studies are required to validate the power and the limitations of such an approach when

44 applied to macro-eukaryotes. Here, we analyzed water sample metagenomes to estimate zooplankton

45 diversity in 22 freshwater lakes across Eastern Canada. We tested the coherence of data based on

46 morphologically identified zooplankton taxa and molecular genetic data derived from shotgun sequencing

47 of environmental DNA collected at the same time. RV coefficients showed a significant correlation

48 between the relative abundance of zooplankton families derived from small subunit rRNA genes extracted

49 from the metagenomes and morphologically identified zooplankton. However, differences in congruence

50 with morphological counts were detected when varied bioinformatic approaches were applied to

51 presence-absence data. This study presents one of the first diversity assessments of a group of aquatic

52 metazoans using metagenomes and validates the coherence of the community composition derived from

53 genetic and classical species surveys. Overall, our results suggest that metagenomics has the potential

54 to be further developed to describe metazoan biodiversity in aquatic ecosystems, and to advance this

55 area we provide key recommendations for workflow improvement. 


\section{Introduction}

57 In the context of intensifying global change, there is a growing need for broad scale monitoring strategies

58 and ecosystem assessment (Cardinale et al., 2012; Cordier et al., 2020). Approaches based on

59 environmental DNA (eDNA), broadly defined as the total pool of DNA that can be isolated from the

60 environment (Taberlet et al., 2012; Pawlowski, 2020; Rodriguez-Ezpeleta et al., 2021), represent high-

61 throughput, cost-effective, non-invasive tools that are being increasingly used in biodiversity monitoring

62 programs (Bohmann et al., 2014; Deiner et al., 2017). One of the most common methods to interpret the eDNA signal from a complex community is marker gene metabarcoding, which allows for multiple taxa to

64 be investigated in a single sequencing experiment (Hajibabaei et al., 2011; Taberlet et al., 2012). This

65 approach has led to numerous successful biodiversity assessments of terrestrial and aquatic biota,

66 including metazoans (e.g. Hänfling et al., 2016; Sigsgaard et al., 2016; Deiner et al., 2017; Taberlet et al.,

67 2018), and has the potential to help us gain a more holistic view of an ecosystem with hundreds of

68 organisms identified simultaneously from one environmental sample. Metabarcoding is a highly sensitive

69 approach that can detect rare or cryptic species (Thomsen et al., 2012; Port et al., 2016), and is seen as

70 a promising approach in ecological assessment studies of aquatic ecosystems (Aylagas et al., 2016;

71 Cordier et al., 2017; Yang and Zhang, 2020). Nevertheless, metabarcoding as well as other PCR-based

72 techniques, such as quantitative PCR, introduce biases. For example, universal primers used to barcode

73 multiple groups of taxa simultaneously do not necessarily bind equally to different templates, leading to

74 amplification bias or the complete loss of certain groups (Tedersoo et al., 2015; Alberdi et al., 2018; Kelly

75 et al., 2019).

76 Metagenomics, broadly defined as the application of high-throughput shotgun sequencing

77 technologies to capture the entire pool of species present in an eDNA sample without targeting a specific

78 gene marker (Tringe and Rubin, 2005), is an emerging approach but to date has been primarily applied to

79 study microbial communities (Grossart et al., 2020). Metagenomics has only recently gained traction in

80 the study of larger organisms such as metazoans and is now seen as a complement (Singer et al., 2020)

81 and potential alternative to metabarcoding. There are several reasons that might explain the low number

82 of studies using metagenomics to investigate eukaryotes (Barnes and Turner, 2016). First, the efficiency

83 of metagenomics to capture the macro-eukaryote signal is not fully understood. Generally, it is believed 
84 that macro-eukaryotes are present in much lower densities in the environment compared to microbes

85 (Azam and Malfatti, 2007), which might limit the recovery of the macro-eukaryote DNA signal. Second,

86 issues related to the large size and low coding density of eukaryote nuclear genomes may contribute to

87 poor recovery of eukaryotes in environmental metagenomes. For example, genomes of eukaryotes

88 contain many repetitive elements that are difficult to assemble into scaffolds, as well as long non-coding

89 sequences which are generally less taxonomically informative (Bik et al., 2012). Abundance estimations

90 of eukaryotes based on shotgun sequencing are further complicated by the high interspecific variability in

91 the number of rRNA gene copies per nucleus (Bik et al., 2012). Finally, both micro- and macro-organisms

92 will often not find a match in reference databases unless they closely relate to an organism that has had

93 its whole genome sequenced. This is a well-known challenge in any eDNA assessments, but curated

94 DNA reference databases are growing rapidly, and thus it is expected that such limitations will continue to

95 decrease in the near future (e.g., Lewin et al. 2018).

96 Despite the challenges, a handful of studies have shown promising results in applying

97 metagenomics for broad biodiversity assessments of metazoans in water (e.g. Cowart et al. (2018);

98 Singer et al.(2020); Machida et al. (2021); Manu et al. (2021)) and sediment samples (e.g. Pedersen et al.

99 (2016); Gelabert et al. (2021)). This type of work, however, requires adapting bioinformatics pipelines to

100 accommodate the diluted metazoan signal in eDNA, especially when targeting rare organisms. For

101 instance, in microbial metagenomic studies, reads are typically assembled before being mapped to

102 genomes for annotation. However, this approach is not always feasible when working with extra-

103 organismal eDNA, likely due to the degraded nature and limited amount of starting genetic material

104 (Barnes and Turner, 2016). An alternative to assembly is to annotate directly via mapping of

105 metagenomic reads to reference databases of nucleotide or proteins sequences. Although

106 computationally intensive, this approach has been reported effective when the output is processed using

107 a Last Common Ancestor (LCA) algorithm or in combination with compositional interpolated Markov

108 models (Quince et al., 2017). Given the relatively nascent nature of this field, we chose to evaluate the

109 differences in diversity detected between targeting a taxonomically informative gene marker, the $18 \mathrm{~S}$

110 rRNA gene in eukaryotes (i.e. SSU rRNA gene approach), vs. a broader analysis of the tens of millions of

111 metagenomic reads (i.e. whole metagenome approach). 
Here, we provide new insight on the effectiveness and reliability of metagenomics applied to extra-

113 organismal eDNA in water samples for describing freshwater zooplankton. Our main questions are: i) can

114 we effectively detect zooplankton diversity in lake water metagenomes, ii) how does the metagenomic

115 gene prediction approach based on a single taxonomic marker (SSU rRNA gene) compare to mapping

116 the entire eukaryotic fraction of metagenome reads, and iii) do diversity metrics derived from

117 metagenomes show similar responses to key environmental gradients as those detected with

118 morphological taxonomic surveys? We assessed zooplankton (Cladocera, Copepoda, and Rotifera)

119 diversity based on surface water metagenomes from 22 lakes in Eastern Canada and compared these

120 results with zooplankton data from morphologically identified samples collected in net hauls from the

121 same sites. Our study is a timely response to the growing interest in adapting metagenomics techniques

122 for advancing a holistic perspective of aquatic food webs across all domains of life from a single

123 environmental snapshot.

125 2. Methods

\section{2.1. Sites description}

127 The 22 lakes were sampled as part of the Natural Sciences and Engineering Research Council of

128 Canada (NSERC) Canadian Lake Pulse Network campaign in summer 2017 (Huot et al., 2019). (Figure

129 1). Lakes span a range of morphological characteristics and trophic status, as summarized in Table S1.

130 Sampling occurred at a station situated at the maximum depth of each lake. The complete field protocol

131 details are provided by LakePulse (NSERC Canadian Lake Pulse Network, 2021).

\section{2.2. Sampling, DNA isolation, and taxonomic identification}

134 2.2.1. Zooplankton morphological identification

135 Crustacean zooplankton were sampled over the depth of the water column from $1 \mathrm{~m}$ above the sediment

136 up to the water surface using a Wisconsin net with $100 \mu \mathrm{m}$ mesh (10 cm net radius and $100 \mathrm{~cm}$ length).

137 For relatively shallow lakes (<6 m-deep), additional vertical hauls were taken in the same manner to

138 increase sample volume. Crustacean zooplankton were anesthetized with $\mathrm{CO}_{2}$ (Alka-Seltzer) and

139 preserved in $70 \%$ ethanol (approx. final concentration) at room temperature. Species-level identification 
140 of crustacean zooplankton was done with a dissecting microscope under $100 x$ to $400 x$ magnification by

141 BSA Environmental Services (Ohio, U.S.A.). Species biomass was estimated following the method from

142 McCauley (1984). A detailed identification protocol is available in Paquette et al. (2021).

143 Rotifer counts were done on Lugol-preserved tow haul samples collected in the same manner as

144 the crustacean zooplankton samples (above), except that instead of sampling from $1 \mathrm{~m}$ above the

145 sediment to the lake surface, the rotifer samples were collected from the euphotic zone only. In several

146 instances, the euphotic zone is identical to max depth minus $1 \mathrm{~m}$. The coherence between the original

147 cladoceran zooplankton counts and the rotifer counts performed on a different set of samples was verified

148 by counting Bosminidae individuals in both sample types, to confirm that the preserved samples for rotifer

149 counting were representative of the original zooplankton samples (Supplementary File 1).

\subsubsection{Environmental DNA sampling for metagenomic analyses}

Water for eDNA was collected at the same station as the net hauls with an acid-washed

152 integrated depth sampler over the euphotic zone down to $2 \mathrm{~m}$ below the surface. Our eDNA sampling

153 strategy aimed at targeting mainly extra-organismal DNA, i.e. DNA that is not contained within whole

154 organisms (Rodriguez-Ezpeleta et al., 2021), sometimes also referred to as 'extracellular DNA' (Taberlet

155 et al., 2012; Bohmann et al., 2014). Thus, for samples dedicated to eDNA analysis, water was first

156 passed through a $100 \mu \mathrm{m}$ nylon mesh to remove large particles, and then up to $500 \mathrm{~mL}$ of water was

157 vacuum-filtered on a Durapore $0.22 \mu \mathrm{m}$ membrane (Sigma-Aldrich, St. Louis, USA) through a glass funnel

158 apparatus at a maximum pressure of $8 \mathrm{inHg}$ until the filter clogged. Filtrations were done on site in a tent,

159 and filters were preserved immediately thereafter in cryovials at $-80^{\circ} \mathrm{C}$ until analysis. Caution was taken to

160 limit foreign DNA contamination in the field. All materials and equipment were acid-washed between

161 lakes, and gloves were worn during sampling and filtering. In the laboratory, DNA was extracted from

162 filters using the DNeasy PowerWater kit (QIAGEN, Hilden, Germany) following the manufacturer's

163 protocol with the addition of two steps as detailed by Garner et al. (2020). DNA was quantified using a

164 Qubit 2.0 fluorometer and the dsDNA BR Assay kit (Invitrogen, Carlsbad, CA, USA). An aliquot of each

165 DNA extract was sent to Genome Quebec facilities (Montreal, Canada) for shotgun library preparation

166 and sequencing on an Illumina NovaSeq 6000 S4 PE150 with flow cell type S2. 


\subsection{Metagenomic analysis pipelines}

169 Raw demultiplexed shotgun sequence files were quality checked using FastQC v.0.11.15. Adapter

170 trimming and quality filtering were done with Trimmomatic v.0.38 (Bolger et al., 2014) with a minimum

171 average quality of 25 and a minimum length of 36 nucleotides. We applied two slightly different

172 approaches to identify eukaryote sequences in the metagenomes (Figure 2).

173 In the whole metagenome approach, all cleaned shotgun paired-end sequences were merged

174 using PEAR (Zhang et al., 2014) before they were aligned against a local database consisting of all

175 Eukarya entries in the NCBI non-redundant nucleotide database with the following parameters: min e-

176 value 0.001 , min percentage identity $=70$, and retaining max 30 hits per read. BLASTn output files were

177 then imported in MEGAN6 v.6.20.17 (Huson et al., 2016) for taxonomic assignment based on the lowest

178 common ancestor (LCA) algorithm with a minimum score of 80 , a minimum similarity of $80 \%$, a minimum

179 support of 2 reads and a minimum complexity filter set at 0.1 . A detailed bioinformatic workflow is

180 available as supplementary material (Supplementary File 2).

181 In the SSU rRNA gene prediction approach (corresponding to 18S rRNA genes in Metazoa), we

182 applied the results of the 'raw reads analysis pipeline' of the European Bioinformatics Institute (EBI)

183 MGnify (Mitchell et al., 2020). The detailed pipeline is described on the EBI website (https://emg-

184 docs.readthedocs.io/en/latest/analysis.html\#raw-reads-analysis-pipeline). Briefly, paired end reads were

185 merged prior to adapter trimming and quality filtering. Additional non-coding RNAs (ncRNAs) were

186 identified with Infernal (Nawrocki and Eddy, 2013) (HHM-only mode) using a library of ribosomal RNA

187 hidden Markov models from Rfam (Kalvari et al., 2018) to identify large and small (LSU and SSU) rRNA

188 genes. Following this, the reads identified as SSU rRNA genes were aligned with BLASTn and annotated

189 following the whole metagenome approach described above (Figure 2).

$191 \quad$ 2.4. Diversity analysis

192 Diversity analyses based on zooplankton assemblages surveyed using both microscopy and

193 metagenomics were conducted in R v.4.1.0. (R Core Team, 2020). All diversity indices were calculated on

194 assemblages binned to the family rank to deal with uneven taxonomic assignment resolution for different

195 zooplankton groups across analytical platforms. The most common diversity metrics (taxonomic richness, 
Shannon index, Pielou's evenness) were estimated on zooplankton abundance data (i.e. the number of

197 individuals per liter or the number of sequencing reads) using the diversity function of the package vegan

198 (Oksanen et al., 2013) and were used in least-square regressions against key environmental gradients

199 identified from an earlier analysis of eastern Canadian LakePulse sites (Griffiths et al., 2021): epilimnetic

200 total phosphorus concentration, specific conductivity, lake depth and an index of watershed disturbance

201 calculated as the human impact index (HI) (Huot et al., 2019). All environmental variables were logarithm

202 transformed, except for $\mathrm{HI}$ values (percentages) that were arcsine transformed.

203 Principal Component Analyses (PCA) were performed separately for each dataset using the

204 function prcomp on both logarithm and Hellinger-transformed abundance (i.e. the number of individuals

205 per liter or the number of reads sequenced) and biomass data where data were available (i.e. only for

206 crustacean zooplankton observations) (Legendre and Gallagher, 2001). The three main principal

207 components were extracted and used to derive an RV coefficient, analogous to Pearson's correlation

208 coefficient for two given multivariate data matrices (Legendre and Birks, 2012). All possible pairwise

209 comparisons between datasets were explored - densities or biomass vs. either SSU rRNA genes or

210 whole metagenome, and SSU rRNA genes vs. whole metagenome. Coefficient significance was verified

211 with the function coeffRV in FactoMineR (Lê et al., 2008). We also considered the congruence between

212 community identifications done for each sample using morphological data and shotgun analyses by

213 calculating pairwise Jaccard and Bray-Curtis dissimilarities (the former based on incidence data and the

214 latter based on relative abundance data (number of individuals per liter) using the function vegdist in

215 vegan (Oksanen et al., 2013). For this analysis, no biomass data was used.

\section{3. Results}

\section{3.1. Zooplankton taxonomy diversity across analytical platforms}

219 Based on the microscopic analyses, we detected an average zooplankton family-level richness of 11.1

220 across the 18 lakes with complete zooplankton counts (Table 1; rotifer data were missing for three lakes).

221 The most dominant families in terms of counts were the Bosminidae, Cyclopidae and Daphniidae,

222 whereas the dominant families in terms of biomass (crustacean zooplankton only) were Daphniidae,

223 Cyclopidae, and Diaptomidae. Across the 22 sites, the crustacean community was relatively even based 
224 on abundance data, with a Pielou's evenness index of 0.67 ( $0=$ no evenness, $1=$ complete evenness).

225 Considering just the crustacean zooplankton families for which there is a larger data set of hundreds of

226 lakes across our four focal ecozones (Paquette et al., 2021), we found a comparable amount of richness

227 and evenness within the range of the key environmental gradients captured by our 22 sites (Table 1).

228 High-throughput sequencing yielded on average $~ 28$ million raw reads per metagenome and the

229 number of reads per sample after quality filtering and merging of the pairs varied between 7 and 29

230 million (Supplementary Figure S2). Overall, the proportion of the merged reads assigned to Eukaryotes

231 ranged between 0.5 and $1.2 \%$ of the total paired reads, with up to $46 \%$ of the eukaryotic reads confidently

232 assigned to Metazoans (Supplementary Figure S2). With the whole metagenome BLAST approach, we

233 detected a slightly greater average family richness of 15.95 (Table 1). Relative to the microscopy dataset

234 we found that the assemblages in our 22 lakes were less even (mean Pielou's evenness = 0.47; Table 1).

235 The dominant taxa in terms of reads were Daphniidae, Diaptomidae and Brachionidae (Rotifera). Using

236 the targeted SSU rRNA gene prediction approach, we detected the lowest average family richness

237 relative to the previous two analytical approaches, with a mean of $5.6(\min =1, \max =10)$. The dominant

238 taxa detected were Diaptomidae, Synchaetidae (Rotifera) and Cyclopidae. Comparing across the

239 different platforms, we found that the SSU rRNA gene prediction approach yielded the lowest family

240 diversity values but evenness estimates that were closer to those generated through the microscopic

241 counts for densities (Table 1, Supplementary figure S3).

\section{3.2. Congruence of morphological and sequencing zooplankton families}

244 We found a nested group of family diversity as we moved from SSU rRNA genes, to microscopy to whole

245 metagenome datasets (Figure 3a). Nineteen out of 23 families that were detected at most sites using the

246 whole metagenome approach were also found in the microscopy dataset of the 22 lakes. Families that

247 were absent in the microscopy but present in the metagenomes are taxa that are often characterized as

248 benthic or littoral associated (i.e. Harpacticidae (copepoda), Chironomidae (Diptera larvae), Adinetidae

249 (Rotifera), and Philodinidae (Rotifera)).

250 Zooplankton family occurrences across lakes were compared between the three analytical

251 platforms (microscopy and two metagenomics approaches) to determine the level of congruence between 
252 survey methods (Figure 3b \& 4). The families Ergasilidae (copepoda), Leptodoridae (cladocera), and

253 Holopediidae (cladocera) were consistently absent at most sites (found only in a single or a few sites),

254 whereas the Calanoida group (copepods - order level; found at 11 sites), Synchaetidae (rotifer; found at

25511 sites), and the Cyclopoida group (copepods - order level; found at 13 of the 19 sites) were the three

256 taxa that were most consistently widely detected across all analytical platforms (Figure 4). It is worth

257 noting, however, that since the Calanoida and Cyclopoida groups were binned at order level, they are

258 likely to comprise more than one Family each. The reason for this grouping was two-fold: firstly, the

259 majority of the genetic reference sequences for these clades were lacking finer taxonomic resolution, and

260 secondly, these groups include nauplii or juvenile stages which could not be assigned to one or the other

261 order in the microscopy data based on morphological observations only.

262 When comparing pairwise taxon occurrences across the three datasets for all zooplankton

263 families and with copepods grouped at order level (Calanoida and Cyclopoida), we found consistent

264 detections in $45 \%$ of cases (either 3 out of 3 or 0 out of 3 detections). When comparing microscopy with

265 either genetic approach, the overall number of dual positive detections was higher between microscopy

266 and whole metagenome datasets, with a total of 34.3\% positive matches across 17 lakes (two lakes

267 missing whole metagenome data were excluded) compared to only $17.3 \%$ positive matches for the

268 comparison with SSU rRNA gene data in 19 lakes (Figure 4).

269 To consider the congruence of the entire assemblage between analytical platforms we calculated

270 dissimilarity indices and RV coefficients. From a taxonomic presence-absence perspective (Jaccard

271 distances), whole metagenomes more strongly reflected the community composition based on

272 zooplankton counts than the SSU rRNA gene data $(p=0.0008)$. In contrast, when zooplankton family

273 relative abundances (Bray-Curtis dissimilarities) were considered, we found that the SSU rRNA gene data

274 performed similarly to the whole genome BLAST $(p=0.8051$; Figure 5).

275 To further explore the congruence between assemblages, we calculated the RV coefficient based

276 on the three main PCA axes and found moderate and significant congruence between SSU rRNA gene

277 and morphology data (Table 2). Results were stronger when relative abundance data were log-

278 transformed to account for potential over-representation of model organisms in the reference databases,

279 which can yield inflated assigned read estimates compared to other taxonomic groups that are poorly 
populated in reference databases. When data were Hellinger-transformed, the RV coefficient between whole metagenome and SSU rRNA gene datasets was also of moderate strength (Table 2).

\subsection{Zooplankton diversity patterns over environmental gradients}

284 We investigated the relationship between Family-level zooplankton diversity metrics (richness and

285 Shannon diversity) and major environmental gradients identified in the eastern ecozones: the human

286 impact index, total phosphorus (TP), specific conductivity and lake depth). Our analyses showed that 287 several consistent relationships were apparent across the analytical platforms. For richness, significant

288 negative relationships were observed between TP and both metagenomic datasets (SSU: $\operatorname{adj} . \mathrm{R}^{2}=0.24$,

$289 p=0.02 ;$ WM: adj. $R^{2}=0.29, p=0.01$ ) (Figure 6 \& Supplementary Table S2). A negative but non-

290 significant relationship ( $p=0.24$ ) was observed between TP and richness derived from morphological

291 identifications. We also detected negative relationships between richness and human impact index, but

292 the fit once again was only significant for the metagenomic datasets (SSU: adj. $\mathrm{R}^{2}=0.23, p=0.022$; WM:

293 adj. $R^{2}=0.33, p=0.006$; Morphology: $\left.\operatorname{adj} R^{2}=0.07, p=0.14\right)$. Finally, a marginally significant relationship

294 (adj. $\mathrm{R}^{2}$ adj. $=0.12, p=0.08$ ) was observed between richness derived from the whole metagenome

295 dataset and specific conductivity.

For Shannon diversity, a significant fit was found between the SSU rRNA gene data and TP (adj.

$297 R^{2}=0.18, p=0.03$ ) and between SSU rRNA data and human impact index (adj. $R^{2}=0.15, p=0.056$ ).

298 The fit between Shannon diversity derived from whole metagenome data and specific conductivity was

299 marginally significant (adj. $\left.R^{2}=0.11 p=0.098\right)$ and no significant relationships emerged with lake depth

300 (Figure 6 \& Supplementary Table S2).

\section{Discussion}

303 Consistent with other comparative analyses between eDNA metagenomics and morphological

304 approaches (Stat et al., 2017; Singer et al., 2020), our results show that the match is not perfect. Overall,

305 we detected modest congruence in taxon relative abundance across platforms and varying levels of

306 congruence between analytical platforms when we considered presence-absence data. Interestingly,

307 diversity metrics across all analytical platforms showed similar responses to epilimnetic phosphorus 
concentration, which is often considered a limiting nutrient in many lakes in Eastern Canada. Many important improvements can be implemented in future metagenomic work to help refine the robustness of

310 this approach applied to metazoan biodiversity eDNA surveys (section 4.3).

\subsection{To what extent do water metagenomes represent zooplankton biodiversity?}

313 Using a variety of statistical approaches, we found that zooplankton communities surveyed using

314 morphological counts and metagenomic analyses were, at best, moderately correlated. While local

315 diversity metrics were similar across platforms, whole metagenome analysis detected the highest

316 richness of zooplankton taxa. It is also informative to compare the strength of our results with other eDNA

317 - morphological comparisons. For example, Keck et al. (2021) conducted a meta-analysis of comparative

318 metabarcoding and morphological studies, and found that eDNA detected significantly more taxa than

319 morphological counts, as eDNA may contain traces of taxa distributed outside of the immediate sampling

320 area. Although no such synthetic analysis has been done from metagenomes, we would expect a similar

321 finding. In our molecular dataset, we found four Families of zooplankton that were not recorded as part of

322 the morphological survey, but these taxa are either generally characterized as benthic or littoral

323 associated so may not have been present as individuals in the immediate sampling area. For instance,

324 the Bdelloid rotifers Adinetidae and Philodinidae, which we only found via the whole metagenome

325 analysis, are typically found to live on plants or debris in waters with dense vegetation and are generally

326 not caught in plankton tows (Wallace and Snell, 2010). Across the metabarcoding and metagenomic

327 literature, many have argued that eDNA approaches are more complementary to morphological

328 approaches rather than directly exchangeable, and the coherence between metagenomic and

329 metabarcoding for eukaryotic diversity surveys needs further detailed investigation (Garlapati et al., 2019;

330 Cordier et al., 2020).

\section{4.2. Shotgun sequencing reveals diversity patterns over broad environmental gradients}

333 To explore diversity patterns over broad environmental gradients and among analytical platforms, 334 richness and Shannon diversity metrics were plotted against epilimnetic total phosphorus (TP), specific 335 conductivity, lake depth, and human impact index estimated in the 22 lakes (Huot et al., 2019). Based on 
336 our preliminary dataset, we found relatively consistent patterns in zooplankton diversity across analytical

337 platforms, indicating that shotgun sequencing shows promise for investigating ecological gradients in

338 freshwater systems. Our findings are consistent with results reported by Singer et al. (2020) from a

339 marine system, where despite revealing contrasting taxonomic diversity, both the metagenomic and

340 metabarcoding data revealed similar ecological patterns, which in turn were useful to infer factors related

341 to the ecosystem health and function.

343 4.3. Limitations to the metagenomic approach and prospects

344 Limitations of eDNA-based approaches have been widely studied, although these have been primarily 345 based on PCR-based approaches. Challenges relate mostly to the availability and quality of eDNA itself

346 in water, whereby investigators have identified the conditions contributing to eDNA degradation (Barnes

347 et al., 2014) or the transport of eDNA over long distances (Deiner and Altermatt, 2014). Although our

348 knowledge of these factors is constantly improving the robustness of eDNA research, there are also other

349 aspects of the workflow - from sampling strategies to bioinformatics - which need to be improved to

350 strengthen metagenomic approaches applied to the study of metazoans in the environment.

\subsubsection{Methodological considerations}

352 Our metagenomes were produced for the primary purpose of examining bacteria and archaea and thus

353 the volume of water filtered was only $250 \mathrm{ml}$ to $500 \mathrm{ml}$, depending on how much water could be passed

354 through the $0.22 \mu \mathrm{m}$ membrane before it clogged (Garner et al., 2020). In contrast, the morphological

355 identification of zooplankton was performed on tow haul samples collected from tens of liters across the

356 full water column that were then concentrated to a few hundred milliliters. Furthermore, because the

357 samples for DNA analysis were collected over the photic zone only, they might not fully represent the

358 rotifer and crustacean zooplankton samples which were collected from below the thermocline and over

359 the full water column, respectively. Overall, we are looking at diverging sampling efforts and distributions

360 across approaches, which may have brought about some of the differences.

$361 \quad$ Filter size selection could also play an influential role and may allow one to filter more water in

362 future studies. We opted for a size selection step where we first pre-filtered water through a $100 \mu \mathrm{m}$

363 mesh, which selected mostly for extra-organismal DNA (i.e. DNA that is no longer found within an 
364 organism, as opposed to organismal DNA) (Rodriguez-Ezpeleta et al., 2021). It is however likely that 365 gametes and other juvenile stages in cladocerans and copepods passed through the $100 \mu \mathrm{m}$ mesh and

366 got caught on the $0.22 \mu \mathrm{m}$ membrane, which may have contributed to the inflated number of reads

367 assigned mainly to Daphniidae and copepods in the whole metagenome dataset. Selecting a filter with a

368 pore size better suited to our target organisms may lead to a better overall coverage by minimizing the

369 allocation of sequencing effort to DNA from non-target microbial taxa. For instance, $0.45 \mu \mathrm{m}$ cellulose

370 nitrate filters have been shown to yield consistent results for fish metabarcoding with high repeatability

371 between filtration replicates (Li et al., 2018). Type of filter, pre-filtration step and pore size have all been

372 identified as factors determining the final yield of eDNA, with differences observed between taxa and

373 systems (Bowers et al., 2021)

$374 \quad$ Filtering larger volumes of water combined with an increased sequencing depth may help yield a

375 higher number of reads and more diversity for Eukarya, which are very much underrepresented in

376 metagenomes in contrast with bacteria and archaea. Similar to earlier research, we found that the

377 proportion of recovered eukaryotes tends to be $<0.5 \%$ of the total read assignments, either with a

378 genome wide approach (Stat et al., 2017; Cowart et al., 2018) or a gene-centric approach (Tedersoo et

379 al., 2015). In contrast, the filtration of $10 \mathrm{~L}$ of water targeting extracellular DNA combined with ultra-deep

380 sequencing was shown to yield 100 million reads per metagenome from a brackish lagoon and improved

381 the coverage for Eukarya to a proportion corresponding to over $4 \%$ of the total number of reads (Manu,

382 2021). Other emerging target enrichment techniques such as hybridization capture have great potential to

383 improve the detection of metazoans in metagenomes (Seeber et al., 2019; Sevigny et al., 2021).

384 Hybridization capture utilizes RNA probes carefully designed to bind the gene region of interest,

385 enhancing the signal of desired taxa without introducing PCR-induced biases. Recent results based on

386 ultra-deep sequencing have shown that the coverage for eukaryotes may be improved when combining

387 shotgun sequencing with DNA target-capture methods (Manu, 2021). Alternatively, metatranscriptomics is

388 an emerging and promising approach for characterizing zooplankton communities. A recent study by

389 Lopez et al. (2021) comparing zooplankton estimates from observational with both amplicon sequencing

390 and metatranscriptomics datasets has revealed higher congruence of observational zooplankton 
391 abundance and composition with metatranscriptomics estimates compared to amplicons sequencing

392 using genomic (gDNA) and complementary DNA (cDNA) amplicons sequencing.

\subsubsection{Bioinformatics considerations}

394 Carefully designed bioinformatic workflows are crucial for robust taxonomic assignment of sequencing

395 reads. Our data suggest that using the whole metagenomic reads can capture the widest pool of

396 biodiversity, but that the taxonomically informative gene markers, such as the SSU rRNA genes in

397 eukaryotes better reflected the observed relative abundance of zooplankton families based on

398 microscopy. We also found that in using a targeted approach using SSU rRNA genes as taxonomic

399 markers, several taxa were missing or did not get taxonomically assigned using the lowest common

400 ancestor (LCA) algorithm, even though they were present in relatively high abundances in microscopy

401 counts. Some taxa were consistently missing or almost absent from our genetic datasets (such as the

402 Bosminidae), despite being one of the most abundant taxonomic groups in microscopy. Such

403 incongruences between traditional and metabarcoding data have frequently been reported (see Keck et

404 al., 2021). We hypothesize that part of the issue with missing taxa in our metagenomes is caused by the

405 same bioinformatic limitations as in any genetic-based study: the current lack of complete reference

406 molecular data limits our ability to assign taxonomy to sequence reads. In molecular datasets, and

407 especially in shotgun sequencing data, a large portion of the reads generated only get assigned to Class

408 level or lower. These reads are typically filtered out bioinformatically, meaning that they are not

409 considered in the estimation of diversity indices or in comparisons with other datasets. Therefore, we

410 might be widely underestimating the abundance and diversity of certain taxonomic groups which are not

411 populated in reference databases.

412 Since using the full metagenome read set is computationally intensive and does not appear to

413 yield a higher correlation with morphology-based identifications, a reasonable compromise that might

414 increase coverage without multiplying computation efforts could be the combination of a few targeted

415 genetic markers. For example, metagenome reads mapped against both the SSU and LSU rRNA gene

416 markers has been shown to improve taxa recovery in a study of marine plankton from DNA preserved in

417 marine sediment (Armbrecht, 2020). In addition to nuclear SSU rRNA genes, we investigated the

418 mitochondrial cytochrome c oxidase subunit I ( $\mathrm{COI}$ ) but found the coverage for this marker to be very low 
419 for metazoans, most likely due to the generally lower cellular abundance of mitochondria compared to

420 ribosomes. For this reason, we did not pursue further metagenomic COI marker analyses.

421 There is clearly an urgent need for curated molecular databases to improve interpretation of

422 eDNA-based molecular datasets. This is especially the case for freshwaters, where monitoring efforts are

423 limited and yet provide habitat for a disproportionate number of taxa per unit area (Strayer and Dudgeon,

424 2010). Currently there are insufficient data for many taxa such that we cannot even assess the state of

$425 \sim 40 \%$ of freshwater species in Canada (Desforges et al., 2021). Initiatives to improve sequencing

426 coverage of eukaryotic biodiversity are underway, including the Barcode of Life Data System (BOLD)

427 (Ratnasingham and Hebert 2007), the International Barcode of Life (IBOL) (https://ibol.org/), the Earth

428 BioGenome Project (https://www.earthbiogenome.org/), i5K for arthropods (Robinson et al., 2011), and

429 Diat.barcode for Diatoms (Rimet et al 2019). Such initiatives will multiply the number of curated

430 references for taxonomic marker genes, which is key to improving taxonomic assignments in eDNA

431 studies.

\section{5. Conclusion}

434 In this study, metagenomics and classical morphological analyses of zooplankton applied to 22

435 freshwater lakes yielded contrasting abundance estimates but comparable diversity assessments at the

436 family level. Metagenomics detected more taxa, including some that generally live outside the pelagic

437 photic zone where the samples were taken, which is to be expected given the persistence and transport

438 of eDNA in nature. Although metagenomic techniques still need to be improved with better adapted

439 sampling protocols and refined bioinformatics pipelines specific to eukaryotic genomes, our results

440 suggest enormous potential for extending metagenome analysis to the investigation of zooplankton and

441 other aquatic micro- and macro-eukaryotes. Our comparative study contributes to a better understanding

442 of how the metagenomic approach might contribute to biodiversity and ecological assessments in

443 complement to other traditional and eDNA approaches.

444

445 List of figures: 
446 Figure 1. Map of Canada showing the location of the 22 lakes in Eastern Canada and their trophic status

447 based on total phosphorus concentration: oligotrophic (less than $10 \mu \mathrm{g} / \mathrm{L})$, mesotrophic $(10-30 \mu \mathrm{g} / \mathrm{L})$ and

448 eutrophic (greater than $30 \mu \mathrm{g} / \mathrm{L}$ ).

449 Figure 2. Detailed bioinformatics workflow used on shotgun sequencing data.

450 Figure 3. a) Diagram showing the number of zooplankton families and their overlap in detection via the

451 three approaches: morphology-based microscopy (blue), whole metagenome sequencing (green), and

452 gene prediction approach based on the small subunit subset of reads (orange). b) Divergent plots

453 showing the number of lakes in which each zooplankton family were detected via the different

454 approaches (grey: microscopic identification; green: whole-metagenome approach; orange: small-subunit

455 (SSU) rRNA genes approach).

456 Figure 4. Heatmap with the number of each zooplankton Family-level (or Order-level in the case of

457 Cyclopoida and Calanoida copepods and unidentified families of Rotifera) detection across analytical

458 platforms (microscopy, whole metagenome, and small subunit (SSU) rRNA genes). A value of zero

459 signifies that a Family/Order was absent from all datasets.

460 Figure 5. Boxplots showing Bray-Curtis (left) and Jaccard (right) dissimilarities between the microscopy-

461 based taxonomic composition and the sequence read composition using the whole-metagenome

462 approach (green) and the SSU rRNA gene subset approach (orange). The significant ANOVA test is

463 indicated with an asterisk.

464 Figure 6. Diversity metrics (left: Taxonomic richness, right: Shannon entropy) estimated from microscopy,

465 SSU rRNA genes (SSU), and whole metagenome (WM) datasets plotted against environmental gradients

466 using generalized additive model (GAM). Environmental data were log transformed, except for the human

467 impact index (expressed as percentage), which was arcsine transformed. Red and yellow backgrounds

468 identify significant and marginally significant relationships, respectively. Adjusted R-squared and $p$-values

469 for each GAM are listed in Supplementary Table S2)

471 Data archiving statement:

472 Sequence data were submitted to the EBI metagenomics platform for analysis and archiving under Study

473 MGYS00003941. 


\section{References}

477 Alberdi, A., Aizpurua, O., Gilbert, M. T. P., and Bohmann, K. (2018). Scrutinizing key steps for reliable metabarcoding of environmental samples. Methods Ecol. Evol. 9, 134-147. doi:10.1111/2041210X.12849.

Armbrecht, L. H. (2020). The potential of sedimentary ancient DNA to reconstruct past ocean ecosystems. Oceanography 33, 116-123. doi:10.5670/oceanog.2020.211. doi:10.3389/fmars.2016.00096.

Azam, F., and Malfatti, F. (2007). Microbial structuring of marine ecosystems. Nat. Rev. Microbiol. 5, 782791. doi:10.1038/nrmicro1747.

Barnes, M. A., and Turner, C. R. (2016). The ecology of environmental DNA and implications for conservation genetics. Conserv. Genet. 17, 1-17. doi:10.1007/s10592-015-0775-4.

Barnes, M. A., Turner, C. R., Jerde, C. L., Renshaw, M. A., Chadderton, W. L., and Lodge, D. M. (2014).

492 Bik, H. M., Porazinska, D. L., Creer, S., Caporaso, J. G., Knight, R., and Thomas, W. K. (2012).

493 Sequencing our way towards understanding global eukaryotic biodiversity. Trends Ecol. Evol. 27, 494 233-243. doi:10.1016/j.tree.2011.11.010.

495 Bohmann, K., Evans, A., Gilbert, M. T. P., Carvalho, G. R., Creer, S., Knapp, M., et al. (2014).

496 Environmental DNA for wildlife biology and biodiversity monitoring. Trends Ecol. Evol. 29, 358-367. doi:10.1016/j.tree.2014.04.003.

498 Bolger, A. M., Lohse, M., and Usadel, B. (2014). Trimmomatic: A flexible trimmer for Illumina sequence 499 data. Bioinformatics 30, 2114-2120. doi:10.1093/bioinformatics/btu170.

500 Bowers, H. A., Pochon, X., von Ammon, U., Gemmell, N., Stanton, J. A. L., Jeunen, G. J., et al. (2021). 501 Towards the optimization of edna/erna sampling technologies for marine biosecurity surveillance. 
Water (Switzerland) 13, 1-24. doi:10.3390/w13081113.

503 Cardinale, B. J., Duffy, J. E., Gonzalez, A., Hooper, D. U., Perrings, C., Venail, P., et al. (2012).

504 Biodiversity loss and its impact on humanity. Nature 486, 59-67. doi:10.1038/nature11148.

505 Cordier, T., Alonso-Sáez, L., Apothéloz-Perret-Gentil, L., Aylagas, E., Bohan, D. A., Bouchez, A., et al.

506 (2020). Ecosystems monitoring powered by environmental genomics: A review of current strategies

507 with an implementation roadmap. Mol. Ecol., 1-22. doi:10.1111/mec.15472.

508 Cordier, T., Esling, P., Lejzerowicz, F., Visco, J., Ouadahi, A., Martins, C., et al. (2017). Predicting the

$509 \quad$ Ecological Quality Status of Marine Environments from eDNA Metabarcoding Data Using

$510 \quad$ Supervised Machine Learning. Environ. Sci. Technol. 51, 9118-9126. doi:10.1021/acs.est.7b01518.

511 Cowart, D. A., Murphy, K. R., and Cheng, C. H. C. (2018). Metagenomic sequencing of environmental DNA reveals marine faunal assemblages from the West Antarctic Peninsula. Mar. Genomics 37,

513 148-160. doi:10.1016/j.margen.2017.11.003.

514 Deiner, K., and Altermatt, F. (2014). Transport distance of invertebrate environmental DNA in a natural river. PLoS One 9, e88786. doi:10.1371/journal.pone.0088786.

516 Deiner, K., Bik, H. M., Mächler, E., Seymour, M., Lacoursière-Roussel, A., Altermatt, F., et al. (2017).

517 Environmental DNA metabarcoding: Transforming how we survey animal and plant communities.

$518 \quad$ Mol. Ecol. 26, 5872-5895. doi:10.1111/mec.14350.

519 Desforges, J. E., Clarke, J., Harmsen, E. J., Jardine, A. M., Robichaud, J. A., Serré, S., et al. (2021). On

520 the alarming state of freshwater biodiversity in Canada. Can. J. Fish. Aquat. Sci. doi:10.1139/cjfas-

$521 \quad 2021-0073$.

522 Garlapati, D., Charankumar, B., Ramu, K., Madeswaran, P., and Ramana Murthy, M. V. (2019). A review 523 on the applications and recent advances in environmental DNA (eDNA) metagenomics. Rev.

524 Environ. Sci. Biotechnol. 18, 389-411. doi:10.1007/s11157-019-09501-4.

525 Garner, R. E., Gregory-Eaves, I., and Walsh, D. A. (2020). Sediment Metagenomes as Time Capsules of $526 \quad$ Lake Microbiomes. mSphere 5, 1-15. doi:10.1128/mSphere.00512-20.

527 Gelabert, P., Sawyer, S., Bergström, A., Margaryan, A., Collin, T. C., Meshveliani, T., et al. (2021). 528 Genome-scale sequencing and analysis of human, wolf, and bison DNA from 25,000-year-old 529 sediment. Curr. Biol. 31, 3564-3574.e9. doi:10.1016/j.cub.2021.06.023. 
Griffiths, K., Jeziorski, A., Paquette, C., Taranu, Z. E., Baud, A., Antoniades, D., et al. (2021). Multi-trophic level responses to environmental stressors over the past $\sim 150$ years: Insights from a lake-rich region of the world. Ecol. Indic. 127, 107700. doi:10.1016/j.ecolind.2021.107700.

533 Grossart, H. P., Massana, R., McMahon, K. D., and Walsh, D. A. (2020). Linking metagenomics to 534 aquatic microbial ecology and biogeochemical cycles. Limnol. Oceanogr. 65, S2-S20.

535 doi:10.1002/Ino.11382.

536 Hajibabaei, M., Shokralla, S., Zhou, X., Singer, G. A. C., and Baird, D. J. (2011). Environmental barcoding: A next-generation sequencing approach for biomonitoring applications using river benthos. PLoS One 6. doi:10.1371/journal.pone.0017497.

Hänfling, B., Handley, L. L., Read, D. S., Hahn, C., Li, J., Nichols, P., et al. (2016). Environmental DNA metabarcoding of lake fish communities reflects long-term data from established survey methods.

542 Huot, Y., Brown, C. A., Potvin, G., Antoniades, D., Baulch, H. M., Beisner, B. E., et al. (2019). The NSERC Canadian Lake Pulse Network: A national assessment of lake health providing science for water management in a changing climate. Sci. Total Environ. 695, 133668. doi:10.1016/j.scitotenv.2019.133668.

Huson, D. H., Beier, S., Flade, I., Górska, A., El-Hadidi, M., Mitra, S., et al. (2016). MEGAN Community

Kalvari, I., Nawrocki, E. P., Argasinska, J., Quinones-Olvera, N., Finn, R. D., Bateman, A., et al. (2018). Non-Coding RNA Analysis Using the Rfam Database. Curr. Protoc. Bioinforma. 62, 1-44.

552 Keck, F., Blackman, R. C., Bossart, R., Brantschen, J., Couton, M., Hürlemann, S., et al. (2021). MetaEdition - Interactive Exploration and Analysis of Large-Scale Microbiome Sequencing Data. PLoS

555 Kelly, R. P., Shelton, A. O., and Gallego, R. (2019). Understanding PCR Processes to Draw Meaningful Conclusions from Environmental DNA Studies. Sci. Rep. 9, 1-14. doi:10.1038/s41598-019-48546-x.

557 Lê, S., Josse, J., and Husson, F. (2008). FactoMineR: An R Package for Multivariate Analysis. J. Stat. 
Softw. 1. Available at: https://www.jstatsoft.org/v025/i01.

Legendre, P., and Birks, H. J. B. (2012). From Classical to Canonical Ordination. doi:10.1007/978-94007-2745-8.

561 Legendre, P., and Gallagher, E. (2001). Ecologically meaningful transformations for ordination of species data. Oecologia 129, 271-280. doi:10.1007/s004420100716.

563 Lewin, H. A., Robinson, G. E., Kress, W. J., Baker, W. J., Coddington, J., Crandall, K. A., et al. (2018).

564 Earth BioGenome Project: Sequencing life for the future of life. Proc. Natl. Acad. Sci. U. S. A. 115, 565 4325-4333. doi:10.1073/pnas.1720115115.

566 Li, J., Lawson Handley, L. J., Read, D. S., and Hänfling, B. (2018). The effect of filtration method on the 567 efficiency of environmental DNA capture and quantification via metabarcoding. Mol. Ecol. Resour. $568 \quad$ 18, 1102-1114. doi:10.1111/1755-0998.12899.

569 Lopez, M. L. D., Lin, Y., Sato, M., Hsieh, C., Shiah, F., and Machida, R. J. (2021). Using

570 metatranscriptomics to estimate the diversity and composition of zooplankton communities. Mol.

$571 \quad$ Ecol. Resour., 1-15. doi:10.1111/1755-0998.13506.

572 Machida, R. J., Kurihara, H., Nakajima, R., Sakamaki, T., Lin, Y.-Y., and Furusawa, K. (2021).

573 Comparative analysis of zooplankton diversities and compositions estimated from complement DNA 574 and genomic DNA amplicons, metatranscriptomics, and morphological identifications. ICES J. Mar. 575 Sci. doi:10.1093/icesjms/fsab084.

576 Manu, S. (2021). A Novel Metagenomic Workflow for Biomonitoring across the Tree of Life using PCR-

577 free Ultra-deep Sequencing of Extracellular eDNA. ARPHA Conf. Abstr. 4, 0-3.

$578 \quad$ doi:10.3897/aca.4.e64864.

579 Mccauley, E. (1984). "The estimation of the abundance and biomass of zooplankton in samples," in $A$ 580 Manual for the Assessment of Secondary Productivity in Fresh Waters, eds. J. A. Downing and F. H. $581 \quad$ Rigler (Blackwell Scientific Publishers), 228-265.

582 Mitchell, A. L., Almeida, A., Beracochea, M., Boland, M., Burgin, J., Cochrane, G., et al. (2020). MGnify:

583 The microbiome analysis resource in 2020. Nucleic Acids Res. 48, D570-D578.

$584 \quad$ doi:10.1093/nar/gkz1035.

585 Nawrocki, E. P., and Eddy, S. R. (2013). Infernal 1.1: 100-fold faster RNA homology searches. 
Bioinformatics 29, 2933-2935. doi:10.1093/bioinformatics/btt509.

587

588

589

590

591

592

593

594

595

596

597

598

599

600

601

602

603

604

605

606

607

608

609

610

611

612

613

Oksanen, J., Blanchet, F. G., Kindt, R., Legendre, P., Minchin, P. R., O'Hara, R. B., et al. (2013). vegan: Community Ecology Package. R package version 2.0-10. http://CRAN.Rproject.org/package=vegan .

Paquette, C., Gregory-Eaves, I., Beisner, B. E., and Heino, J. (2021). Multi-scale biodiversity analyses identify the importance of continental watersheds in shaping lake zooplankton biogeography. J. Biogeogr., jbi.14153. doi:10.1111/jbi.14153.

Pawlowski, J. (2020). eDNA: what's behind the term? preprint. doi:10.1111/mec.15643.

Pedersen, M. W., Ruter, A., Schweger, C., Friebe, H., Staff, R. A., Kjeldsen, K. K., et al. (2016). Postglacial viability and colonization in North America's ice-free corridor. Nature 537, 45-49. doi:10.1038/nature19085.

Port, J. A., O’Donnell, J. L., Romero-Maraccini, O. C., Leary, P. R., Litvin, S. Y., Nickols, K. J., et al. (2016). Assessing vertebrate biodiversity in a kelp forest ecosystem using environmental DNA. Mol. Ecol. 25, 527-541. doi:10.1111/mec.13481.

Quince, C., Walker, A. W., Simpson, J. T., Loman, N. J., and Segata, N. (2017). Shotgun metagenomics, from sampling to analysis. Nat. Biotechnol. 35, 833-844. doi:10.1038/nbt.3935.

R Core Team (2020). R: A language and environment for statistical computing. Vienna, Austria: R Foundation for Statistical Computing Available at: http://www.r-project.org/.

Robinson, G. E., Hackett, K. J., Purcell-Miramontes, M., Brown, S. J., Evans, J. D., Goldsmith, M. R., et al. (2011). Creating a Buzz About Insect Genomes. Science (80-. ). 331, 1386-1388.

Rodriguez-Ezpeleta, N., Morissette, O., Bean, C. W., Manu, S., Banerjee, P., Lacoursière-Roussel, A., et al. (2021). Trade-offs between reducing complex terminology and producing accurate interpretations from environmental DNA: Comment on "Environmental DNA: What's behind the term?" by Pawlowski et al., (2020). Mol. Ecol. 2021, 1-5. doi:10.1111/mec.15942.

Seeber, P. A., McEwen, G. K., Löber, U., Förster, D. W., East, M. L., Melzheimer, J., et al. (2019). Terrestrial mammal surveillance using hybridization capture of environmental DNA from African waterholes. Mol. Ecol. Resour. 19, 1486-1496. doi:10.1111/1755-0998.13069.

Sevigny, J., Leasi, F., Simpson, S., Di Domenico, M., Jörger, K. M., Norenburg, J. L., et al. (2021). Target 
enrichment of metazoan mitochondrial DNA with hybridization capture probes. Ecol. Indic. 121, 106973. doi:10.1016/j.ecolind.2020.106973.

616 Sigsgaard, E. E., Nielsen, I. B., Bach, S. S., Lorenzen, E. D., Robinson, D. P., Knudsen, S. W., et al. (2016). Population characteristics of a large whale shark aggregation inferred from seawater environmental DNA. Nat. Ecol. Evol. 1, 0004. doi:10.1038/s41559-016-0004.

Singer, G. A. C., Shekarriz, S., McCarthy, A., Fahner, N., and Hajibabaei, M. (2020). The utility of a metagenomics approach for marine biomonitoring. bioRxiv, 2020.03.16.993667.

Stat, M., Huggett, M. J., Bernasconi, R., Dibattista, J. D., Berry, T. E., Newman, S. J., et al. (2017). Ecosystem biomonitoring with eDNA: Metabarcoding across the tree of life in a tropical marine environment. Sci. Rep. 7, 1-11. doi:10.1038/s41598-017-12501-5.

Strayer, D. L., and Dudgeon, D. (2010). Freshwater biodiversity conservation: Recent progress and future challenges. J. North Am. Benthol. Soc. 29, 344-358. doi:10.1899/08-171.1.

Taberlet, P., Bonin, A., Zinger, L., and Coissac, E. (2018). "Environmental DNA: For biodiversity research and monitoring," in Environmental DNA: For Biodiversity Research and Monitoring, 121-126. doi:10.1093/oso/9780198767220.001.0001.

Taberlet, P., Coissac, E., Hajibabaei, M., and Rieseberg, L. H. (2012). Environmental DNA. Mol. Ecol. 21,

632 Tedersoo, L., Anslan, S., Bahram, M., Põlme, S., Riit, T., Liiv, I., et al. (2015). Shotgun metagenomes and multiple primer pair-barcode combinations of amplicons reveal biases in metabarcoding analyses of

635 Thomsen, P. F., Kielgast, J., Iversen, L. L., Wiuf, C., Rasmussen, M., Gilbert, M. T. P., et al. (2012). Monitoring endangered freshwater biodiversity using environmental DNA. Mol. Ecol. 21, 2565-2573.

638 Tringe, S. G., and Rubin, E. M. (2005). Metagenomics: DNA sequencing of environmental samples. Nat. Rev. Genet. 6, 805-14. doi:10.1038/nrg1709. E. M-P. Varin, M. Beaulieu, \& Y. Huot doi:10.17118/11143/18662. 
642 Wallace, R. L., and Snell, T. W. (2010). Rotifera. Ecol. Classif. North Am. Freshw. Invertebr., 173-235.

643 doi:10.1016/B978-0-12-374855-3.00008-X.

644 Yang, J., and Zhang, X. (2020). eDNA metabarcoding in zooplankton improves the ecological status

645 assessment of aquatic ecosystems. Environ. Int. 134, 105230. doi:10.1016/j.envint.2019.105230.

646 Zhang, J., Kobert, K., Flouri, T., and Stamatakis, A. (2014). PEAR: A fast and accurate Illumina Paired-

647 End reAd mergeR. Bioinformatics 30, 614-620. doi:10.1093/bioinformatics/btt593.

648 
649 Table 1. Summary of the main diversity indices (mean (min - max)) estimated for the three survey

650 approaches at the Family taxonomic level. Microscopic estimates for Shannon and Pielou were based on

651 abundance data (the number of individuals per liter, or the number of sequenced reads).

\begin{tabular}{lccc}
\hline Approach & $\begin{array}{c}\text { Taxonomic } \\
\text { richness }\end{array}$ & Shannon index & Pielou's evenness \\
\hline Microscopy & $11.1(7-15)$ & $1.6(0.7-1.9)$ & 0.67 \\
SSU rRNA gene subset & $5.6(1-10)$ & $1.2(0-1.9)$ & 0.74 \\
Whole metagenome & $14.2(10-21)$ & $1.3(0-2.0)$ & 0.47 \\
Microscopy (198 LakePulse sites) & $6.49(2-10)$ & $1.14(0.1-1.8)$ & 0.61 \\
\hline
\end{tabular}

652

653

654 
655 Table 2. Results of the congruence test between datasets (RV coefficients with significance values) using

656 two types of data transformation (Hellinger or $\log _{10}+1$ ) on genetic and morphological data (abundance of

657 all zooplankton and biomass of crustacean zooplankton only).

\begin{tabular}{lllcc}
\hline Matrices compared & Taxa included & Transformation & $\begin{array}{c}\text { RV } \\
\text { coefficient }\end{array}$ & p-value \\
\hline Density-SSU & All zooplankton & Hellinger & $\mathbf{0 . 3 9}$ & $\mathbf{0 . 0 0 4}$ \\
Density-WM & All zooplankton & Hellinger & 0.006 & 0.93 \\
SSU-WM & All zooplankton & Hellinger & $\mathbf{0 . 3 8}$ & $\mathbf{0 . 0 0 3}$ \\
\hline Density-SSU & All zooplankton & Log $_{10}+1$ & $\mathbf{0 . 4 8}$ & $\mathbf{0 . 0 0 0 6}$ \\
Density-WM & All zooplankton & Log $_{10}+1$ & 0.12 & 0.39 \\
SSU-WM & All zooplankton & Log $_{10^{+}}+1$ & 0.17 & 0.19 \\
\hline Biomass-SSU & Crustaceans only & Hellinger & 0.21 & 0.12 \\
Biomass-WM & Crustaceans only & Hellinger & 0.03 & 0.96 \\
Biomass-Density & Crustaceans only & Hellinger & $\mathbf{0 . 6 4}$ & $\mathbf{3 . 3 3 E - 0 6}$ \\
SSU-WM & Crustaceans only & Hellinger & $\mathbf{0 . 2 5}$ & $\mathbf{0 . 0 4 5}$ \\
\hline Biomass-SSU & Crustaceans only & Log $_{10}+1$ & $\mathrm{NS}$ & $\mathrm{NS}$ \\
Biomass-WM & Crustaceans only & Log $_{10}+1$ & $\mathrm{NS}$ & $\mathrm{NS}$ \\
Biomass-Density & Crustaceans only & Log $_{10}+1$ & $\mathbf{0 . 5 1}$ & $\mathbf{0 . 0 0 0 2}$ \\
SSU-WM & Crustaceans only & Log $_{10}+1$ & 0.12 & 0.28 \\
\hline Density-SSU & Rotifers only & Hellinger & 0.09 & 0.68 \\
Density-WM & Rotifers only & Hellinger & 0.13 & 0.53 \\
SSU-WM & Rotifers only & Hellinger & 0.09 & 0.70 \\
\hline Density-SSU & Rotifers only & Log $_{10}+1$ & 0.08 & 0.96 \\
Density-WM & Rotifers only & Log $_{10}+1$ & 0.10 & 0.66 \\
SSU-WM & Rotifers only & Log $_{10}+1$ & 0.02 & 0.96 \\
\hline
\end{tabular}


bioRxiv preprint doi: https://doi.org/10.1101/2021.10.27.465999; this version posted October 28, 2021. The copyright holder for this preprint (which was not certified by peer review) is the author/funder, who has granted bioRxiv a license to display the preprint in perpetuity. It is made available under aCC-BY-NC-ND 4.0 International license.

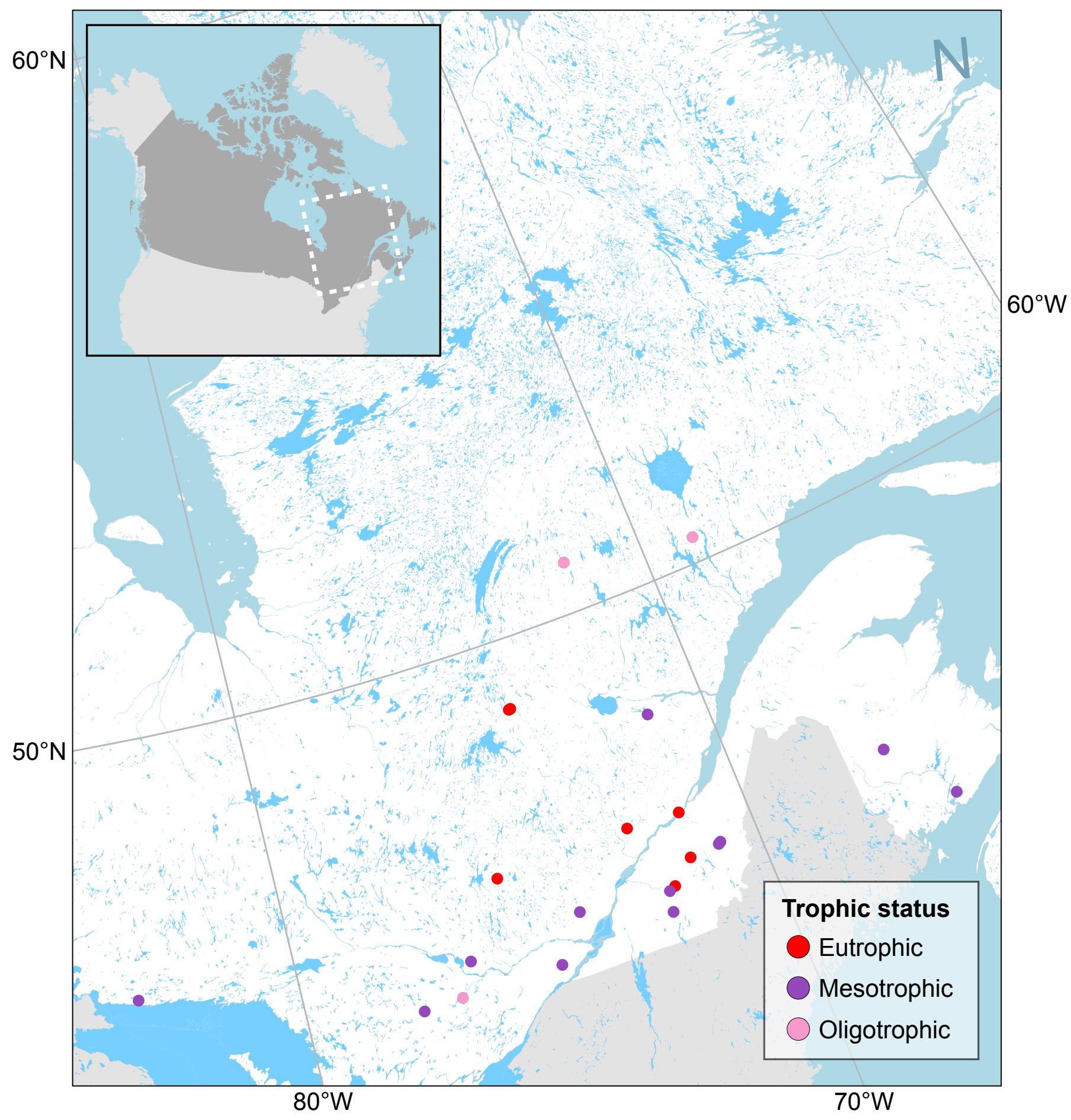




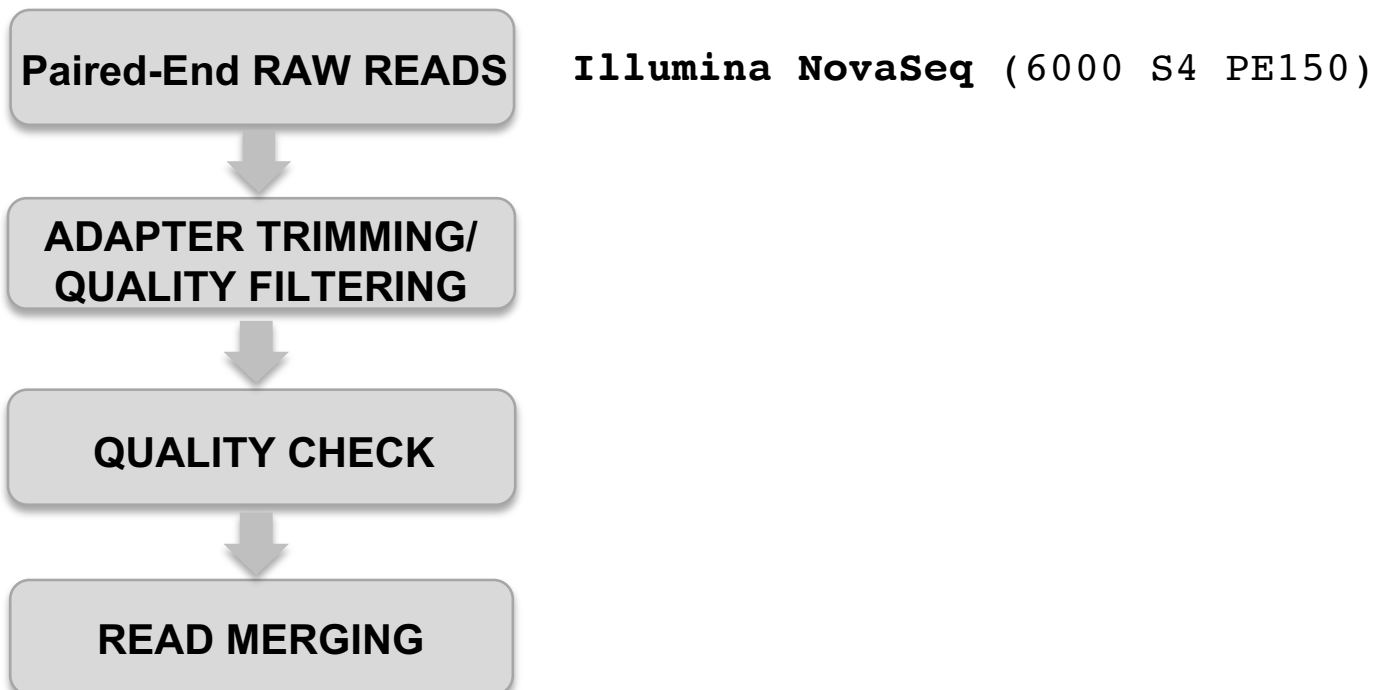

READ MAPPING \& TAXONOMIC ASSIGNMENT

\section{Whole metagenome}

BLAST against

Eukaryota db

\section{Gene prediction}

Extraction of SSU rRNA genes
BLAST against

Eukaryota db 

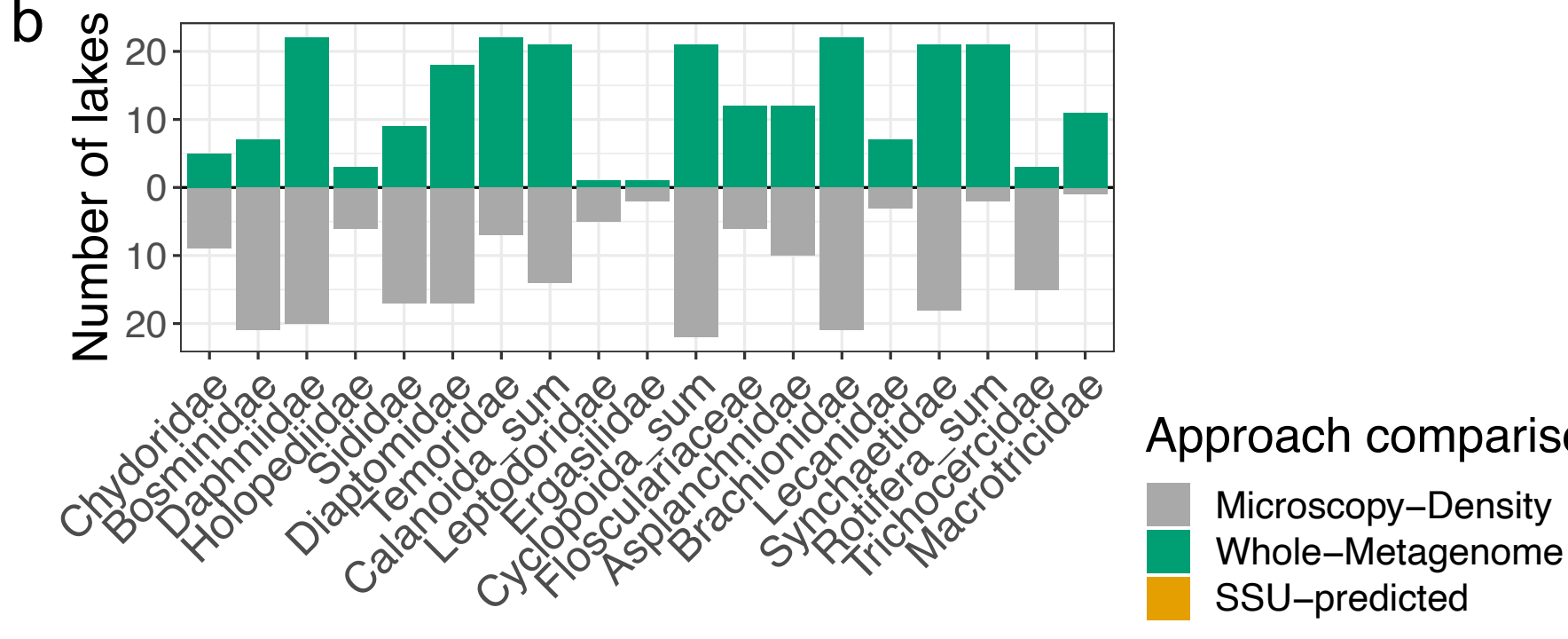

Holopediidae

Sididae

Chaoboridae

Leptodoridae

Diaptomidae Calanoida (O.)

Temoridae

Cyclopoida (O.)

Ergasilidae

Lecanidae

Trichocercidae

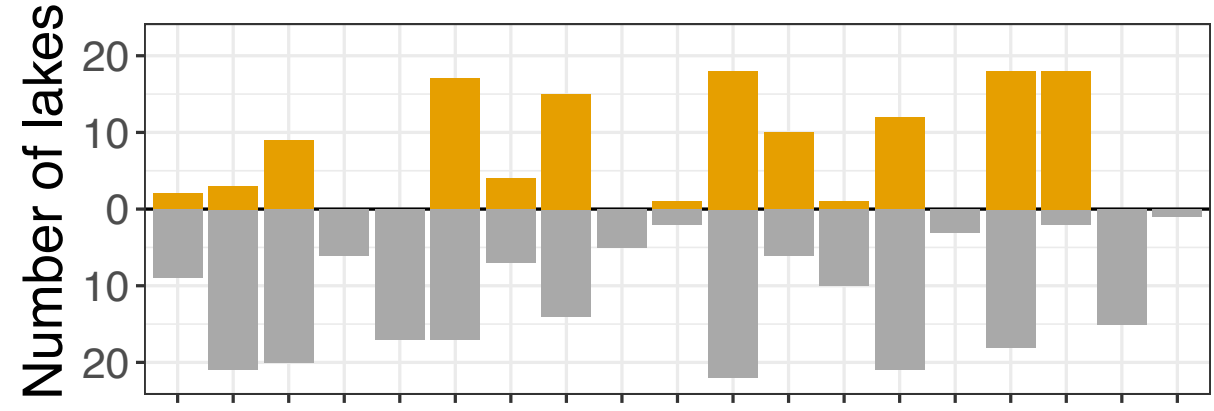

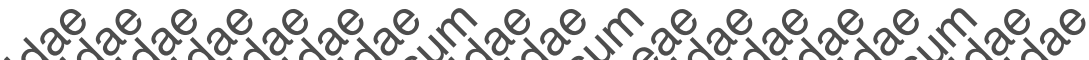

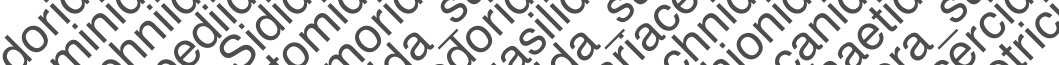

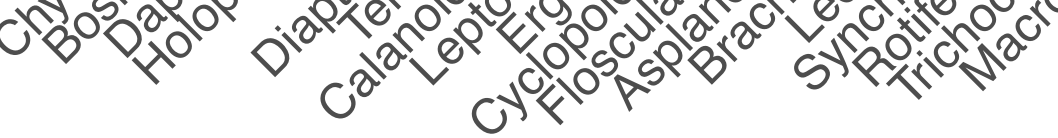

Family 


\section{Occurences of zooplankton families across 3 platforms}
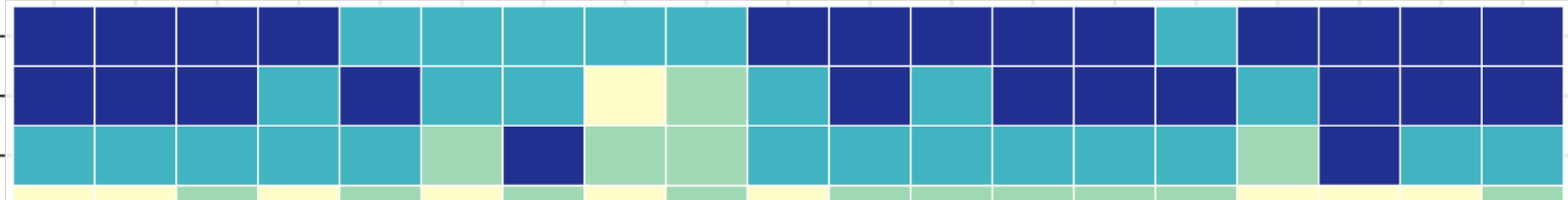

Macrotricidae -

Trichocercidae -

Synchaetidae

Lecanidae -

Brachionidae -

Asplanchnidae -

Flosculariaceae -

Philodinidae -

Adinetidae -
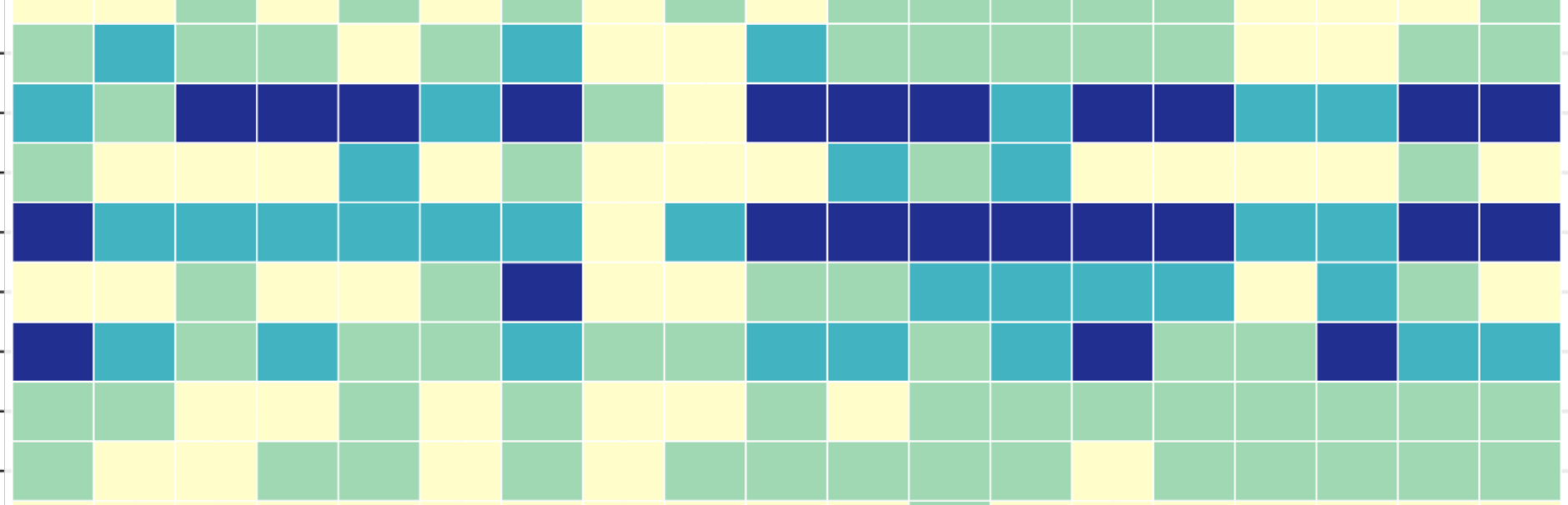

Ergasilidae -

Leptodoridae -

Chaoboridae -

Chironomidae -

Harpacticidae -

Temoridae -

Diaptomidae

Sididae -

Holopediidae -

Daphniidae -

Bosminidae -

Chydoridae
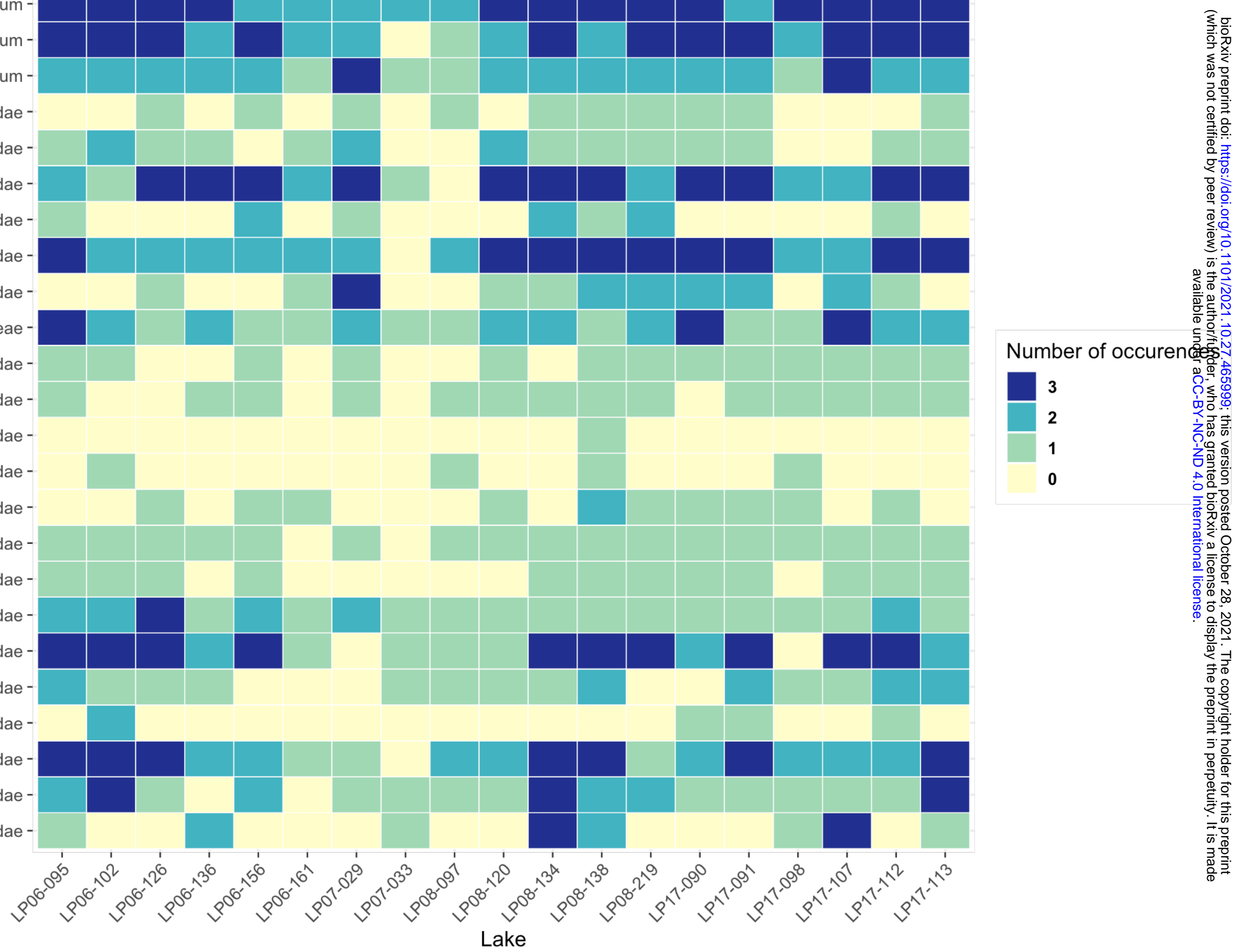

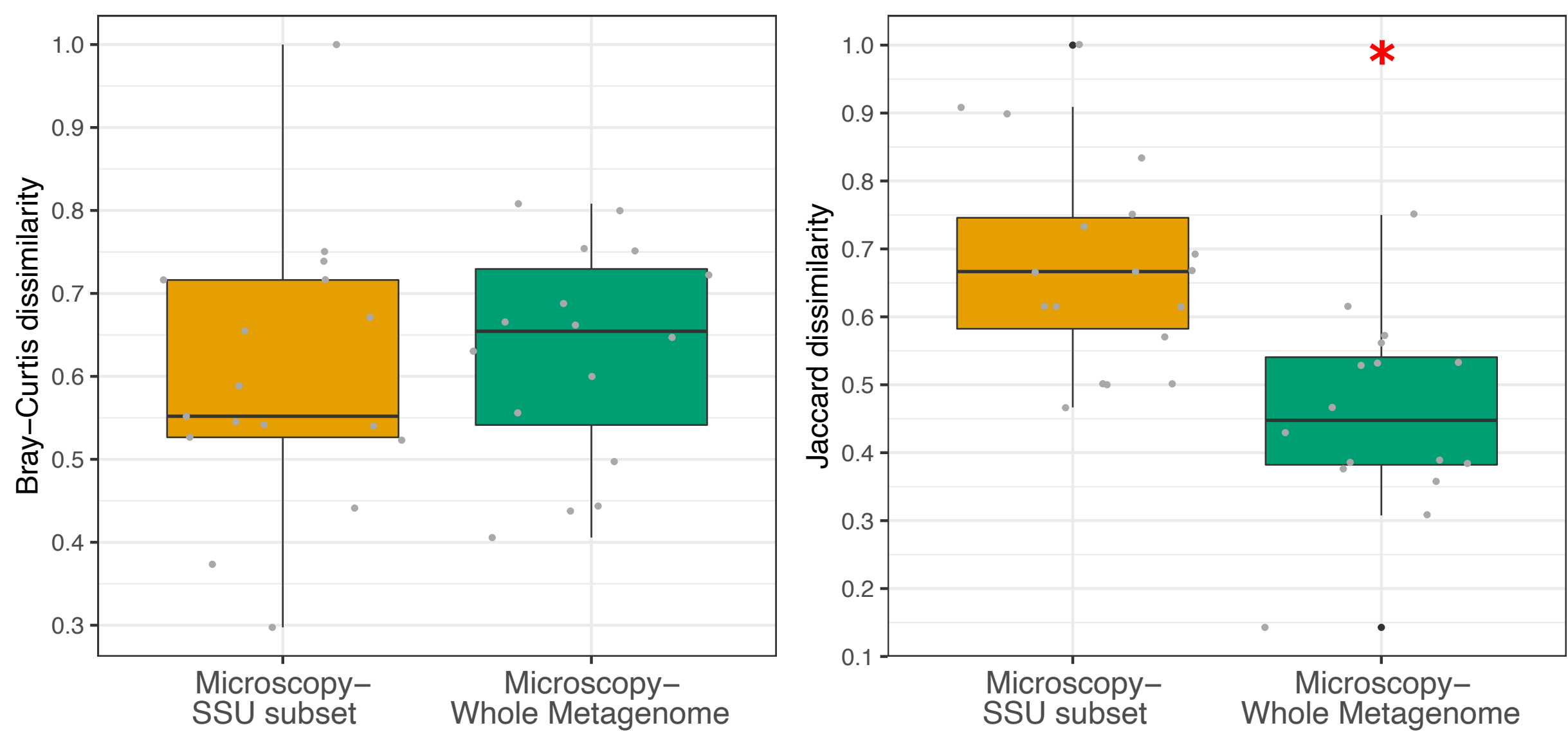
